\title{
Quels apports éducatifs du jeu vidéo Minecraft en éducation? Résultats d'une recherche exploratoire menée auprès de 118 élèves du primaire
}

\author{
What are the educational outcomes of \\ using the Minecraft video game in education? \\ Results from an exploratory study conducted \\ with 118 elementary school students.
}

Thierry Karsenti

Université de Montréal

Julien Bugmann

Université de Montréal

doi:10.18162/fp.2018.459

\section{ésumé}

L'objectif de cette recherche est d'identifier les usages et les avantages de l'intégration scolaire du jeu Minecraft à l'école. Cette recherche a été menée auprès de 118 élèves du primaire. Dix instruments de collecte des données ont été utilisés. Ce projet a permis aux élèves d'optimiser les avantages éducatifs d'un usage scolaire et encadré de Minecraft. Cet usage pédagogique de Minecraft à l'école, créé pour cette recherche exploratoire, a notamment permis de mettre en évidence différents apprentissages attendus en contexte scolaire et faisant partie, pour certains, des compétences à maîtriser par les élèves au XXle siècle : hausse de la motivation, développement de la collaboration, apprentissage de la programmation, développement de compétences informatiques.

\section{Mots-clés}

Jeux vidéo, apprentissage, codage, école primaire

\section{Abstract}

The aim of this project is to identify the uses and advantages of the scholastic integration of the game Minecraft. This study was conducted with 118 elementary school students, which allowed them to reap the benefits of a supported and purposeful use of Minecraft. Overall, ten data collection tools

were used throughout the study. The supported gameplay, created specifically for this research project, revealed multiple positive outcomes to the use of this video game in a scholastic setting, including: increased motivation, learning of computer programming, development of computer science skills.

Keywords Video game, learning, coding, primary school.

\section{Introduction}

Minecraft est le deuxième jeu vidéo le plus vendu de tous les temps ${ }^{1}$. De surcroît, il est actuellement utilisé dans de plus en plus d'écoles aux États-Unis ${ }^{2}$, en Suède ${ }^{3}$, et même au Canada. Dans un contexte où de plus en plus d'écoles se servent du jeu vidéo Minecraft pour instruire les élèves, il nous est apparu important d'apporter un éclairage scientifique sur la question du potentiel scolaire d'un tel jeu vidéo, afin de mieux comprendre ses impacts éducatifs auprès des jeunes. Concrètement, nous avons décidé de nous pencher sur la question dans le cadre d'une recherche exploratoire dont les principaux objectifs étaient de mettre en évidence les principaux usages du jeu vidéo Minecraft à l'école et de relever les avantages liés à la pratique du jeu vidéo Minecraft à l'école.

\section{Des jeux vidéo pour apprendre... à l'école}

Pourquoi s'intéresser au jeu vidéo à l'école? Tout simplement parce que l'importance du jeu en éducation est, depuis longtemps, valorisée par de nombreux auteurs (Dewey et Deledalle, 1983; Piaget, 1959; Winnicott, 1975) et qu'aujourd'hui, le recours à sa forme numérique, le jeu vidéo, qui plus est, première industrie culturelle au monde ${ }^{4}$, apparaît donc comme une évidence. Cependant, tout ne fut pas aussi évident et simple concernant le recours au jeu vidéo en salle de classe, même si la recherche a montré qu'y avoir recours pouvait offrir des conditions très clairement favorables à certains apprentissages (Baranowski et al., 2003) et produire des effets positifs sur les joueurs aux niveaux « cognitifs, affectifs et psychomoteurs " (Shaftel, Pass et Schnabel, 2005). En effet, il a été prouvé que la prise de plaisir suscitée par le fait de jouer aux jeux vidéo était particulièrement importante dans tout apprentissage (Picard, 2016), au même titre 
que la situation de flow (ou d'expérience optimale) (Csikszentmihalyi, 1990) que peut faire naître cette pratique. Dans ce contexte de flow, toute l'attention du joueur est concentrée sur la tâche en cours. Il est alors pleinement dédié à l'environnement qu'il découvre, qu'il expérimente, et il est donc ouvert aux apprentissages. La possibilité d'un décrochage, d'une démotivation, d'une perte de sens est ici éloignée, et cette distance peut amener l'apprenant à des découvertes et à une focalisation cognitive forte sur le contenu proposé. Le recours au jeu vidéo répond, de surcroît, aux besoins futurs des jeunes élèves, qui vont devoir maîtriser de nouvelles compétences dans les prochaines années, des compétences dites du $21^{\mathrm{e}}$ siècle (Fonction publique de l'Ontario, 2016). Au Québec, cela est d'autant plus important lorsque l'on sait que près de $15 \%$ des élèves sortent sans aucun diplôme ni qualification du système scolaire (Ministère de l'Éducation et de l'Enseignement supérieur, 2017). Mais quel jeu utiliser pour intéresser les élèves, les « immerger » et surtout développer leurs apprentissages? Nous proposons pour cela d'avoir recours au jeu vidéo Minecraft.

\section{Le jeu de bac à sable Minecraft pour « construire » ses apprentissages}

Certains jeux vidéo, tels que Minecraft, amèneraient les élèves à développer des compétences en résolution de problèmes, en travail collaboratif, mais pourraient aussi stimuler leur motivation. C'est le constat effectué par Méndez, Arrieta, Dios, Encinas et Queiruga-Dios (2016) qui ont analysé la pratique du jeu vidéo par des étudiants en architecture. Aussi, selon Callaghan (2016), l'utilisation de Minecraft en contexte éducatif permet le développement d'apprentissages, notamment en ce qui concerne l'engagement, la collaboration ou encore la créativité. Minecraft est un jeu vidéo qui possède de nombreuses similitudes avec les Lego et qui pourrait presque correspondre à son versant numérique. Alors qu'avec les Lego, les utilisateurs peuvent déplacer des cubes, produire et reproduire des constructions en tous genres, ils peuvent faire de même avec Minecraft, mais cette fois-ci dans un univers virtuel, constitué de cubes pixélisés et où tout objet ou composant sera sous cette forme. Ce jeu stimule également la motivation des utilisateurs qui, dans le mode créatif, améliorent leurs compétences en résolution de problèmes (Thorsteinsson et Niculescu, 2016). Les auteurs soulignent également le fort potentiel du jeu pour les enseignants, grâce notamment à l'amplitude créative qu'il permet. Notre intérêt se porte aussi et surtout sur l'étude du comportement des élèves en session de jeu, tout particulièrement en ce qui concerne le recours à un jeu vidéo pour lequel la recherche a montré un intérêt majeur pour les élèves. En effet, certains auteurs vont jusqu'à parler d'un impact «immense » de Minecraft en recherche, mais aussi en éducation, particulièrement grâce à l'apprentissage par le jeu, la création ou encore l'enseignement par les autres qu'il rend possible (Nebel, Schneider et Rey, 2016). Nous nous intéressons particulièrement pour cette étude à la motivation intrinsèque des élèves au sens où l'entendent Deci et Ryan (1985) et qui implique la pratique d'une activité par des individus, et en l'occurrence ici des élèves, car ils en retirent du plaisir et de la satisfaction. Aussi, alors que l'on aborde de plus en plus la question des compétences du $21^{\mathrm{e}}$ siècle, et leur importance pour les jeunes générations (Fonction publique de l'Ontario, 2016), il apparaît que la pratique du jeu vidéo Minecraft pourrait mettre en pratique certaines de ces compétences, mais aussi améliorer celles en littératie médiatique des apprenants après seulement six mois de pratique du jeu vidéo (Morgan, 2015). Certains projets intégrant ce jeu vidéo s'intègrent même dans le monde réel et vont jusqu'à devenir des propositions architecturales complètes. En témoignent les écrits de Magnussen et Elming (2015) qui font état de

projets de refonte de certains quartiers de la ville de Copenhague par des étudiants qui ont remodélisé 
certains quartiers, le tout en collaboration avec les personnels de la ville. Ce jeu permettrait donc l'apprentissage de la technologie, du travail d'équipe et de la construction (Overby et Jones, 2015), grâce notamment à la créativité qu'il permet de stimuler chez les élèves (Moffat, Crombie et Shabalina, 2017). Il représente finalement une opportunité unique pour les joueurs de se montrer créatifs et de comprendre des concepts plus aisément réalisables d'une manière virtuelle que dans la vie réelle (Cipollone, Schifter et Moffat, 2014). Ce jeu permettrait donc le développement d'apprentissages scolaires, ce qui permettrait de répondre à notre premier sous-objectif, mais il serait aussi susceptible de développer des apprentissages et des aptitudes extrascolaires.

Certaines études ont aussi montré que Minecraft avait un fort potentiel en termes de développement de la collaboration, de connectivité sociale, mais aussi et surtout en tant qu'outil éducatif (Riordan et Scarf, 2016) au point de voir un impact positif du jeu vidéo Minecraft chez des personnes atteintes de troubles du spectre autistique (TSA). Ce jeu, qui n'a ni but ni objectif, permettrait en effet aux joueurs de se plonger dans leur propre récit, de créer et d'explorer (Riordan et Scarf, 2016). On retrouve même un réseau dédié à la pratique du jeu vidéo Minecraft par des enfants atteints de TSA (http://www. autcraft.com) et qui peut participer au développement d'une nouvelle forme de socialité pour ces participants (Ringland, Wolf, Faucett, Dombrowski et Hayes, 2016). Pour ces raisons, le jeu vidéo Minecraft est parfois utilisé dans des établissements scolaires afin d'accompagner un enseignement traditionnel, ou encore d'appuyer certains aspects historiques, comme en fait état Craft (2016). Il s'avère même que ce jeu dit de « bac à sable » peut améliorer la maîtrise de l'information des adolescents grâce aux espaces d'apprentissages informels qu'il pousse à côtoyer (Bebbington et Vellino, 2015). Le recours à MinecraftEdu, la toute première version éducative du jeu, a aussi stimulé l'intérêt des élèves pour les sciences et pour l'utilisation des technologies de l'information et de la communication (TIC) en classe (Pusey et Pusey, 2015).

Devant le potentiel du jeu vidéo Minecraft, une version adaptée à l'école, Minecraft Education Edition a vu le jour à la fin de l'année 2016. Il s'agit finalement d'une version de Minecraft pensée et développée pour le milieu éducatif, dans la mesure où elle a été conçue en collaboration avec des enseignants, afin d'aider au mieux les élèves devenus joueurs à acquérir certains apprentissages. Parmi les intérêts aux usages de Minecraft Education Edition mis en avant par le développeur du jeu, Microsoft (en collaboration avec Mojang $\mathrm{AB}$ ), on retrouve le fait d'attirer l'attention des élèves, d'encourager leur créativité, mais aussi de développer la collaboration entre les apprenants.

\section{Vers la construction d'un programme scolaire intégrant Minecraft}

Ainsi, le recours aux jeux vidéo pour l'apprentissage semble prendre de plus en plus de sens dans le domaine éducatif, et les initiatives intégrant ces outils se multiplient, elles aussi. Le jeu vidéo Minecraft, comme nous venons de l'aborder, semble particulièrement pertinent de par l'intérêt qu'il suscite chez les joueurs, mais aussi par son potentiel créatif. Ce jeu, dans un monde ouvert, permettrait aux utilisateurs de construire tout ce qu'ils souhaitent, et à contrario, de tout détruire par la suite... avec une seule limite : leur imagination. On peut ainsi jouer avec l'eau, le feu, la terre, les arbres, etc. sans risque, et en totale liberté. Toutefois, il ne s'agit bien souvent que d'initiatives isolées de la part d'enseignants ou de chercheurs qui développent des projets à court terme et non pas de programmes d'intégration complets de ce jeu vidéo en classe. Par ailleurs, ces initiatives ont rarement lieu au niveau primaire, 
mais davantage au niveau secondaire et ne ciblent pas d'élèves ayant des besoins particuliers. Comme nous l'avons vu, le jeu vidéo peut être utilisé avec succès en contexte scolaire, et de multiples bénéfices sont relevés chez les joueurs. Cependant, aucune recherche ne présente l'intégration d'un " programme scolaire » complet intégrant Minecraft et qui propose un usage pédagogique, mais ludique, dans un cadre normé, et encore moins auprès d'élèves de primaire ayant des difficultés d'apprentissage.

Aussi, à l'ère des compétences du $\mathrm{XXI}^{\mathrm{e}}$ siècle, du tout numérique et de la ludification de la société, il nous apparaît extrêmement important que d'interroger les potentiels effets, sur des élèves en école primaire, de l'intégration d'un programme complet intégrant le jeu vidéo Minecraft en classe. Ce questionnement principal, qui introduit notre objectif d'intégration du jeu vidéo Minecraft en contexte scolaire, est alimenté par deux sous-objectifs, qui sont les suivants : de mettre en évidence les principaux usages du jeu vidéo Minecraft à l'école et de relever les avantages liés à la pratique du jeu vidéo Minecraft à l'école.

\section{Méthodologie}

Nous avons choisi de mettre en place une recherche exploratoire (Trudel, Simard et Vonarx, 2006), parce que ce type de recherche peut être un préalable « à des recherches qui, pour se déployer, s'appuient sur un minimum de connaissances » (p. 39). En fait, la recherche exploratoire permettrait avant tout de baliser une réalité encore peu étudiée. Autrement dit, la recherche exploratoire servirait à produire des connaissances sur des phénomènes inconnus ou nouveaux (Trudel et al., 2006).

En lien avec nos objectifs de recherche, cette section présente la méthodologie de recherche. Comme le préconise la 6 e édition du Publication manual of the American Psychological Association (2013), on y retrouve la présentation des participants, des instruments de collecte de données et des stratégies d'analyse des données recueillies. Une partie sur les forces et les limites méthodologiques de cette recherche vient clore cette section.

\section{Participants}

Ce sont en tout 118 élèves (63 filles, soit 53,4\% des participants, et 55 garçons, soit 46,6 \% des participants) qui ont pris part à cette recherche. Les participants étaient âgés de 9 à 12 ans, avec un âge moyen de 11,3 ans. Tous les participants étaient des élèves d'écoles primaires de la région de Montréal (Canada), dans des milieux où l'indice de défavorisation ${ }^{5}$ variait de 7 à 10 (10 étant attribué aux milieux socioéconomiquement les plus défavorisés). Les participants à cette étude ont été choisis sur une base volontaire, avec le consentement de leurs parents et de l'école. Les données de cette enquête ont été recueillies au cours de l'année scolaire 2016-2017.

\section{Dispositif expérimental mis en place : le programme « scolaire » Minecraft}

Dans le cadre de cette recherche, et pour baliser l'usage scolaire de Minecraft, nous avons procédé à la création d'un programme scolaire : Devenez le maître Minecraft (Figure 1). Ce programme est notamment constitué de 30 tâches scolaires, qui font appel à diverses compétences ou connaissances, 
et qui sont regroupées en 10 niveaux, du plus simple au plus complexe (Figure 1). Ces niveaux permettaient de découvrir progressivement l'utilisation du jeu vidéo Minecraft et d'aller vers une maîtrise accrue du jeu en fin de parcours. Ainsi, le premier niveau amenait les élèves à lancer le jeu en personnalisant leur partie, puis ils étaient amenés, dans le niveau suivant, à se déplacer dans le jeu, puis, graduellement, à améliorer leur maîtrise de l'environnement vidéoludique, etc. Ces étapes, et surtout l'idée de progression, étaient pour nous primordiales dans la compréhension du fonctionnement de l'environnement informatique et du jeu vidéo Minecraft par les élèves.

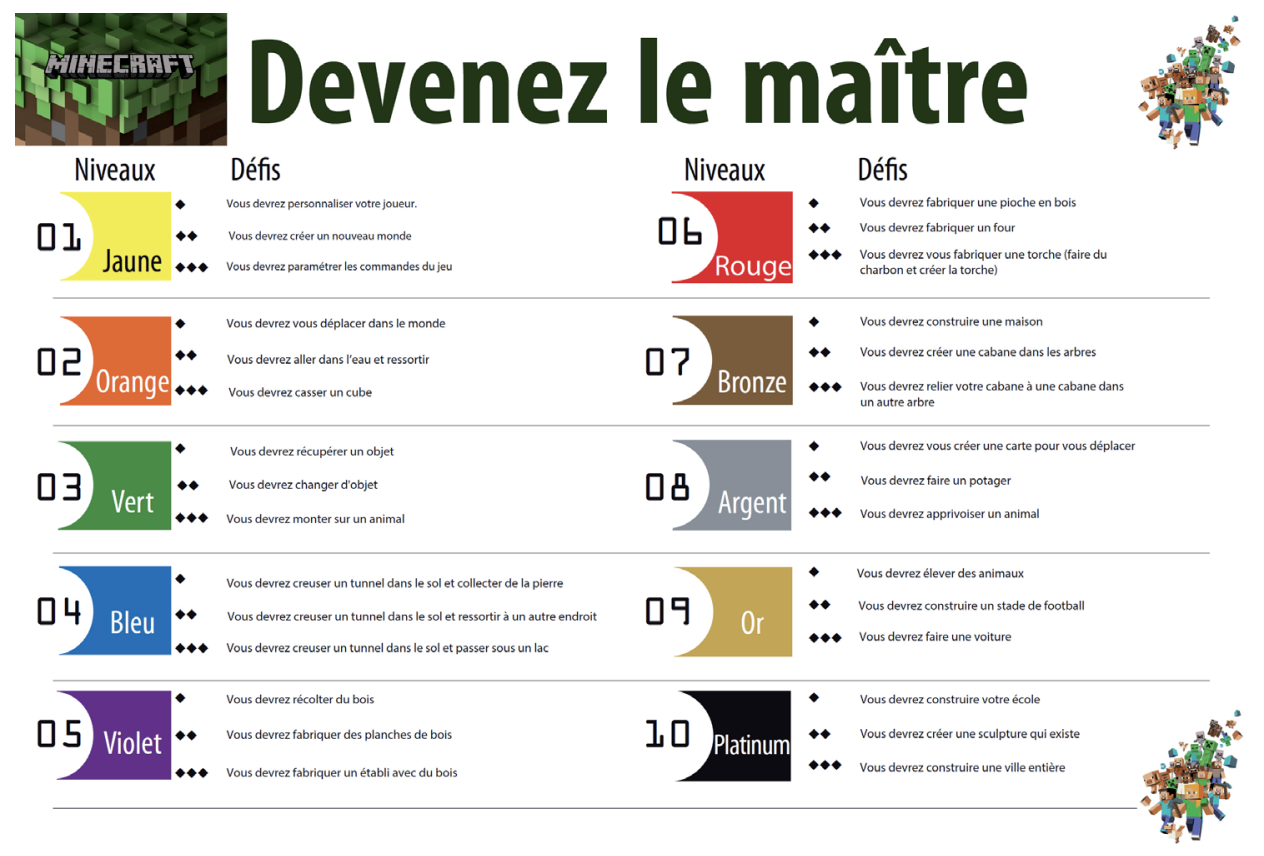

\section{Figure 1}

Niveaux Maître Minecraft.

Afin de stimuler les élèves dans l'atteinte des niveaux supérieurs, nous avons créé des codes de couleurs et des titres pour chacun des niveaux : « Maître Minecraft Jaune Niveau 1 », « Maître Minecraft Orange Niveau 2 », etc. jusqu'au niveau ultime, le niveau « Maître Minecraft Platinum Niveau 10 » pour l'étape la plus élevée. Aussi, comme chaque niveau proposait trois tâches à effectuer, chacune devait être validée par un animateur qui devait attester du niveau accompli sur la fiche individuelle Attestation de passage de niveau Minecraft remise à chaque élève (Figure 2). Les élèves devaient donc effectuer 30 activités au total pour atteindre le niveau le plus élevé et devenir ainsi un Maître Minecraft. 


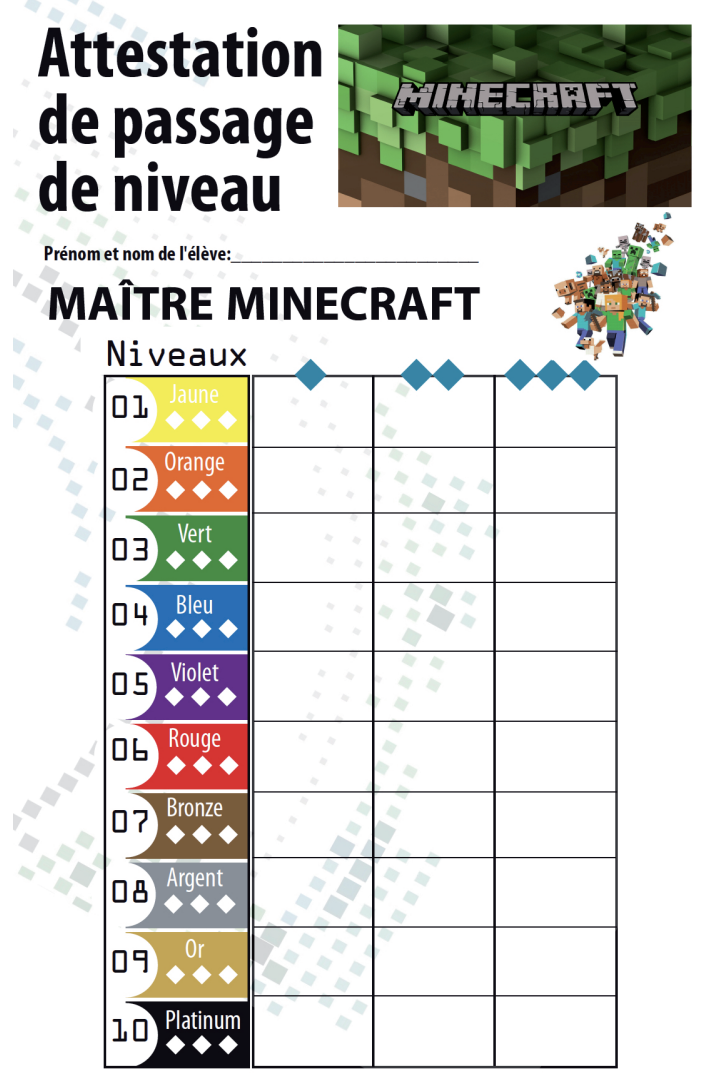

Figure 2

Fiche d'attestation de passage des niveaux du programme scolaire : Devenez le maître Minecraft.

Par ailleurs, toujours pour stimuler les élèves à s'engager dans cet usage encadré du jeu Minecraft, nous avons introduit des bracelets de couleur « Maître Minecraft » pour chacun des niveaux proposés. Ces bracelets étaient remis aux élèves dès lors qu'ils validaient un niveau. Soulignons également que pour certifier leur passage au niveau supérieur, un animateur expert de Minecraft, futur ingénieur, devait valider les différentes étapes réalisées par les élèves. Ce n'est qu'à ce moment-là qu'ils obtenaient un bracelet sur lequel apparaissait le nom et la couleur du niveau atteint, ainsi que des visuels Minecraft. Ces bracelets, rappelons-le, avaient pour objectif de motiver les élèves et de les amener à aller le plus loin possible dans la réalisation des activités proposées dans ce dispositif. Il semble important de rappeler que le rôle de l'animateur fut extrêmement important dans cette recherche exploratoire, puisque c'est lui qui intervenait auprès de l'ensemble des élèves participant à l'activité Minecraft. Le journal de bord qu'il devait remplir à chacune des séances nous a permis de mieux comprendre ce qu'il avait observé. 
Enfin, pour la suite du projet, et pour les élèves qui atteignaient le statut de « Maître Minecraft », nous avons aussi créé des niveaux supérieurs, appelés « Minecraft PRO ». Ces niveaux demandaient des compétences encore plus complexes (Figure 3).

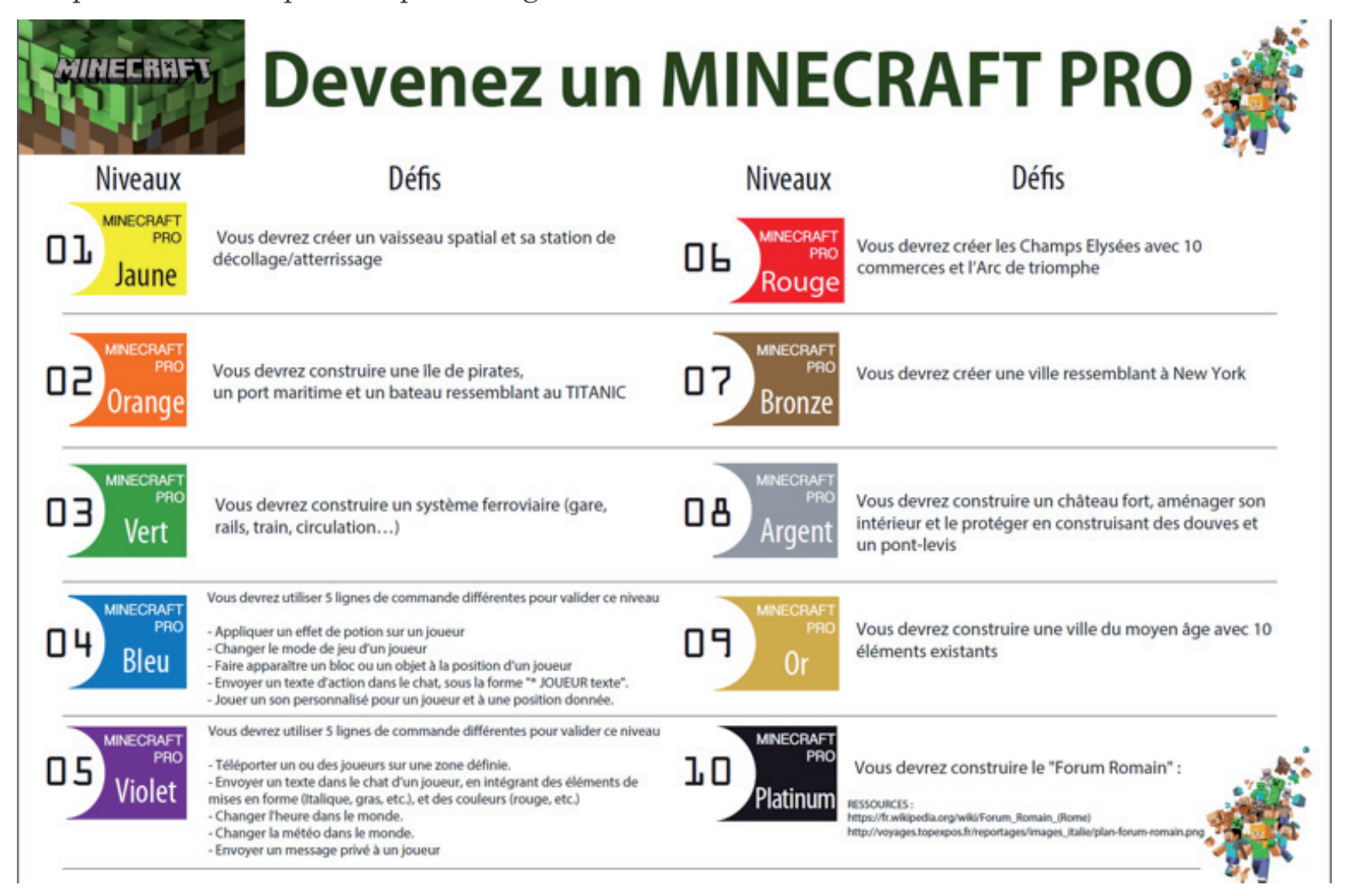

Figure 3

Niveaux Minecraft PRO.

\section{Instruments de collecte de données}

Dans le cadre de cette recherche, 10 méthodes de collecte de données ont été utilisées (Tableau 1). Pourquoi autant de méthodes de collecte de données? Parce que la recherche exploratoire peut également permettre de tester et de choisir une méthode de recherche adéquate comme le confirment Trudel et al. (2006) : «La recherche exploratoire permettrait ainsi de baliser une réalité à étudier ou de choisir les méthodes de collecte des données les plus appropriées pour documenter les aspects de cette réalité » (p.39). 


\section{Tableau 1}

10 méthodes de collecte de données utilisées auprès des participants.

\section{Outil de collecte des données Fréquence Type de données collectées}

\begin{tabular}{lll}
\hline $\begin{array}{l}\text { Questionnaires d'enquêtes auprès des } \\
\text { élèves }\end{array}$ & $\begin{array}{l}\mathrm{n}=4 \text { questionnaires } \\
\text { auprès de } 118 \text { élèves }\end{array}$ & $\begin{array}{l}\text { Données qualitatives et quantitatives sur les } \\
\text { habitudes vidéoludiques des élèves et l'effet } \\
\text { du programme sur leur plaisir en classe et leur } \\
\text { motivation }\end{array}$ \\
\hline $\begin{array}{l}\text { Entrevues individuelles semi-dirigées en } \\
\text { dehors des périodes de jeu supervisé }\end{array}$ & $\begin{array}{l}\mathrm{n}=6 \text { entrevues } \\
\text { de } 30 \text { minutes }\end{array}$ & $\begin{array}{l}\text { Données qualitatives permettant d'interroger la } \\
\text { motivation des élèves }\end{array}$ \\
\hline $\begin{array}{l}\text { Entrevues individuelles brèves auprès de } \\
\text { l'ensemble des élèves participants, lors } \\
\text { des périodes de jeu supervisé }\end{array}$ & $\begin{array}{l}\mathrm{n}=118 \text { entrevues } \\
\text { de } 5 \text { minutes }\end{array}$ & $\begin{array}{l}\text { Données qualitatives permettant d'interroger } \\
\text { la motivation des élèves et d'étudier leurs } \\
\text { comportements en phase de jeu permettant } \\
\text { notamment aux élèves d'exprimer l'effet du jeu sur } \\
\text { leur motivation (Deci et Ryan, 1985) }\end{array}$ \\
\hline $\begin{array}{l}\text { Entrevues de groupe avec des élèves lors } \\
\text { des périodes de jeu supervisé }\end{array}$ & $\mathrm{n}=3$ entrevues &
\end{tabular}

\begin{tabular}{lll}
\hline $\begin{array}{l}\text { Observations vidéographiées des périodes } \\
\text { de jeu supervisé }\end{array}$ & $\begin{array}{l}n=6 \text { observations } \\
\text { filmées de } 75 \text { minutes }\end{array}$ & $\begin{array}{l}\text { Étude du comportement des élèves en session de jeu } \\
\text { et mesure de leur engagement dans la tâche }\end{array}$ \\
\hline $\begin{array}{l}\text { Observations vidéographiées en mode } \\
\text { «think aloud » (Roussel, 2017) d'élèves } \\
\text { qui jouent à Minecraft }\end{array}$ & $\begin{array}{l}n=3 \text { observations de } \\
30 \text { minutes }\end{array}$ & $\begin{array}{l}\text { Étude du comportement des élèves en session de } \\
\text { jeu et confrontation avec leur perception de leurs } \\
\text { activités en jeu }\end{array}$ \\
\hline $\begin{array}{l}\text { Entrevues individuelles avec l'animateur } \\
\text { durant les périodes de jeu supervisé }\end{array}$ & $n=6$ entrevues & $\begin{array}{l}\text { Données qualitatives permettant d'interroger } \\
\text { l'engagement des élèves dans le programme scolaire } \\
\text { Minecraft afin de recueillir son ressenti par rapport à } \\
\text { l'engagement et à la motivation des élèves }\end{array}$
\end{tabular}

\begin{tabular}{lll}
\hline $\begin{array}{l}\text { Suivi des niveaux de jeu atteints par } \\
\text { l'ensemble des élèves participants }\end{array}$ & $\begin{array}{l}n=99 \text { fiches de suivi } \\
\text { des niveaux }\end{array}$ & $\begin{array}{l}\text { Données quantitatives permettant d'interroger } \\
\text { la progression des élèves dans le programme } \\
\text { Minecraft et leur développement de compétences en } \\
\text { programmation }\end{array}$
\end{tabular}

\begin{tabular}{lll}
\hline $\begin{array}{l}\text { Journal de bord hebdomadaire tenu par } \\
\text { l'animateur de l'atelier Minecraft }\end{array}$ & $\begin{array}{l}\mathrm{n}=14 \text { entrées de la part } \\
\text { de l'animateur }\end{array}$ & $\begin{array}{l}\text { Données qualitatives permettant d'évaluer } \\
\text { l'engagement des élèves dans la tâche et données } \\
\text { quantitatives (présence au cours, notamment) }\end{array}$ \\
\hline $\begin{array}{l}\text { "Traces informatiques ", soit les } \\
\text { productions ou créations réalisées par les } \\
\text { élèves à l'aide de Minecraft" }\end{array}$ & $\begin{array}{l}\mathrm{n}=300 \text { visuels (photos } \\
\text { et captures d'écran) }\end{array}$ & $\begin{array}{l}\text { Données qualitatives issues des traces informatiques } \\
\text { (voir Jaillet et Larose, 2009) permettant d'évaluer } \\
\text { la créativité des élèves et les liens avec les attentes } \\
\text { ministérielles proposées dans le programme } \\
\text { Minecraft }\end{array}$
\end{tabular}




\section{Traitement et analyse des données}

Les données recueillies à l'aide du questionnaire comprennent à la fois des échelles de Likert et des réponses ouvertes. Par conséquent, l'analyse qui en ressort est dite de type mixte. L'analyse quantitative comprend des statistiques descriptives élaborées à l'aide du logiciel SPSS $23^{7}$ et de l'outil de sondages en ligne SurveyMonkey ${ }^{8}$. Ces premiers résultats d'analyse sont approfondis et appuyés par une analyse qualitative des réponses ouvertes aux questionnaires, effectuée à l'aide du logiciel QDA Miner'. Elle consistera en une analyse de contenu (L'Écuyer, 1990; Miles et Huberman, 1991) dont le codage semi-ouvert a été construit à partir des réponses des participants en lien avec les principaux objets de recherche (usages et avantages).

L'analyse des données des entrevues individuelles et de groupe s'est inspirée des démarches proposées par L'Écuyer (1990) et Miles et Huberman (1991). Nous avons privilégié une approche de type « analyse de contenu ». Les analyses qualitatives ont à nouveau été facilitées par l'emploi du logiciel QDA Miner, abondamment utilisé dans l'analyse de données qualitatives en recherche (Komis, Depover et Karsenti, 2013).

\section{Forces et limites méthodologiques}

L'une des principales forces de la présente étude réside assurément dans la méthodologie de recherche particulière utilisée. Jumeler des questionnaires d'enquête en ligne à des entrevues individuelles, à des entrevues collectives (pendant ou en dehors des sessions de jeu), à des observations vidéographiées, à des relevés de journal de bord, à un suivi des niveaux de jeu, à l'analyse des traces, de même qu'à des entrevues de type "think aloud", semble constituer, en soi, un avantage majeur pour enrichir et trianguler les résultats obtenus. Les choix méthodologiques effectués ne sont pourtant pas sans limites. Tout d'abord, le fait de travailler à partir des perceptions peut constituer une limite que nous avons tenté de pallier par un vaste échantillon de participants $(n=118)$ et par des méthodes de collecte de données variées, dont des observations vidéographiées. Pour réduire ce biais méthodologique, les analyses effectuées ont systématiquement comparé les réponses des différents types de répondants, mettant en exergue leurs points de divergence lorsque nécessaire.

Une autre limite de l'étude est liée à l'échantillon des participants qui n'était pas aléatoire. Notre choix des participants n'avait pas pour objectif de représenter un sous-ensemble de la population interrogée (les élèves en école primaire à Montréal). En effet, dans notre contexte éducatif, il semblait particulièrement difficile, voire impossible, de procéder à une sélection aléatoire des participants. Nous avons plutôt misé sur un échantillon de convenance, soit un échantillon non probabiliste qui n'aspire pas à être représentatif, mais simplement à utiliser les répondants disponibles ou volontaires et aisément interrogeables. La seule restriction au niveau des participants était qu'ils devaient participer à des périodes de jeu supervisées (Minecraft). 


\section{Principaux résultats}

Les principaux résultats sont présentés en fonction de nos objectifs de recherche, soit :

a) Mettre en évidence les principaux usages du jeu vidéo Minecraft à l'école;

b) Relever les avantages liés à la pratique du jeu vidéo Minecraft à l'école.

Nous illustrons tout d'abord ce projet en mettant en valeur certaines copies d'écran et photos des productions des élèves, commentées brièvement. Puis, nous présentons les principaux impacts éducatifs du jeu Minecraft supervisé en contexte scolaire.

\section{Exemples de productions des élèves}

Dans le cadre de cette étude exploratoire, nous avons recueilli de nombreuses copies d'écrans lorsque les élèves participaient aux sessions de jeu encadré Minecraft. En lien avec le concept de traces informatiques (voir Jaillet et Larose, 2009), il nous a semblé important d'en présenter certaines afin de mieux prendre conscience de l'ampleur des réalisations des élèves. Nous présentons par exemple certaines productions des élèves (Figure 4 et Figure 5) qui illustrent leur créativité, leur engagement pour les tâches demandées, de même que leur maîtrise du jeu Minecraft.

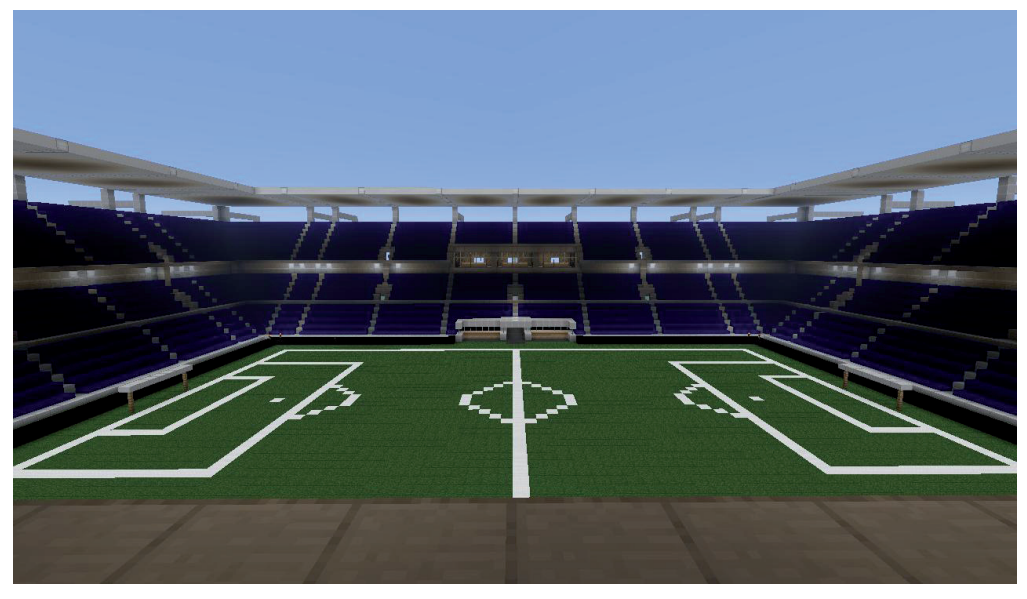

Figure 4

Stade de soccer réalisé par des élèves. 


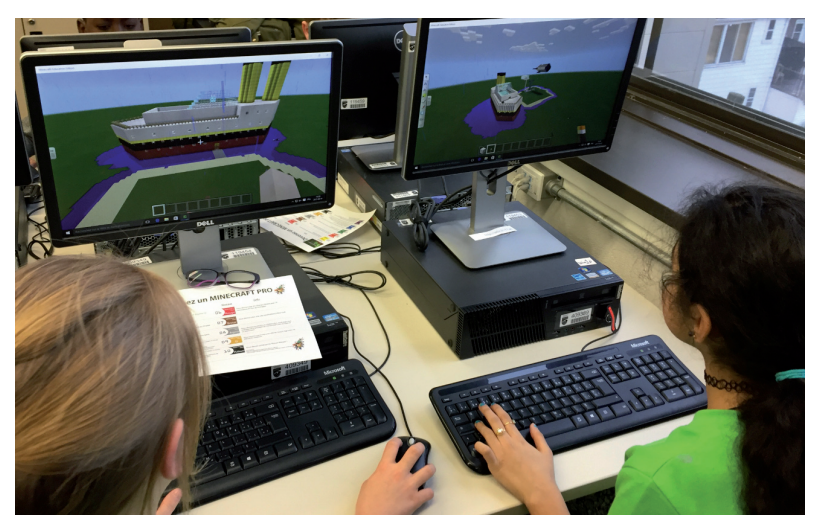

Figure 5

Deux élèves travaillant à la construction du Titanic.

\section{Quels impacts éducatifs pour Minecraft?}

L'analyse des données recueillies lors de cette étude exploratoire a permis de mettre en évidence un grand nombre d'avantages éducatifs liés à l'usage du jeu vidéo Minecraft en classe que nous présentons ici.

\section{Des effets bénéfiques sur la motivation des élèves}

Les données recueillies à l'aide des divers instruments de collecte de données montrent que la participation au jeu Minecraft a un impact direct sur la motivation des élèves, comme l'illustre le commentaire d'un père de famille, dans un courriel adressé à la directrice d'une des écoles. Il y explique qu'il est venu chercher sa fille, mais que cette dernière ne souhaitait pas quitter l'école, car elle peut jouer à Minecraft, alors que la fin des classes est depuis longtemps passée. De plus, même si la participation au projet Minecraft était facultative et se déroulait après l'école, très peu d'absences ont été constatées suite aux relevés effectués par l'animateur. Selon lui, les élèves étaient ainsi «très motivés » et ont montré «beaucoup d'intérêt pour l'atelier Minecraft ». Il rappelle d'ailleurs que « c'est une activité qui est facultative et qu'ils viennent à l'école parce quills ont envie de venir à l'école ». La directrice d'une des écoles a même été contrainte de refuser des élèves à plusieurs reprises, par manque de places, tant il y avait de demandes pour assister à l'atelier Minecraft. Le stimulus « jeu vidéo Minecraft » a donc produit une réaction positive et permettant de maintenir un engagement fort dans la tâche et une motivation continue pour le programme Minecraft. Le fort engagement des élèves dans les tâches proposées, de par leur «immersion » forte dans le jeu (Csikszentmihalyi, 1990), semble avoir facilité ces comportements positifs et facilitateurs d'apprentissages.

L'enquête par questionnaire révèle même que, pour 77,1\% des élèves, le fait de jouer à Minecraft à l'école leur plaisait "énormément». Ce constat est également ressorti des entrevues réalisées avec eux :

- " c'est pas réel, c'est cool, on peut construire des choses»

- "j'aime ça construire des villes»

- "j'aime faire des constructions»

- "Minecraft, comparé aux autres jeux cubiques, c’est vraiment le jeu le plus intéressant » 
- "j’aime créer, faire des maisons, des piscines, etc. "

- «j'aime beaucoup jouer à Minecraft »

- Minecraft, c'est « amusant et en même temps, c'est éducatif»

- "on joue en s'amusant, mais en s'amusant, on apprend des choses"

Cela montre aussi que les motivations des élèves à jouer aux jeux vidéo sont multiples, comme en faisaient état Ferguson et Olson (2013), qui parlent de diverses motivations dans le fait de jouer aux jeux vidéo, par exemple le plaisir ou la réduction du stress en jouant. Certains apprécient le fait d'être libres dans le jeu; d'autres, d'apprendre des choses; d'autres encore, de pouvoir créer des bâtiments. Parmi celles-ci, on retrouve donc la motivation liée à l'apprentissage par le jeu, ce qui rejoint les conclusions de multiples auteurs (Dewey et Deledalle, 1983; Piaget, 1959; Winnicott, 1975). Cela confirme aussi l'intérêt d'un tel jeu vidéo créatif pour les joueurs (Callaghan, 2016). La pratique de ce jeu vidéo a donc participé au développement de la motivation intrinsèque (Deci et Ryan, 1985) des élèves dans la mesure où ces derniers ont pris du plaisir et ont déclaré entre autres « aimer jouer » à Minecraft.

\section{Un suivi des niveaux tout à fait bénéfique}

Les élèves ont, d'une manière générale, suivi les niveaux qui leur ont été proposés tout au long de l'atelier. Ils arrivaient à progresser rapidement, au point d'atteindre, pour certains, le dixième niveau, soit le plus complexe, au bout de quelques séances seulement (c'est le cas de près de $19 \%$ des élèves). L'animateur confirme d'ailleurs cette tendance dès la quatrième séance : «les élèves suivent presque tous les niveaux et au moins la moitié de la classe a dépassé le niveau 7, beaucoup ont fini le niveau 9 ». Le jeu a d'ailleurs été, pour une majorité des élèves, maîtrisé très rapidement. Au bout d'une séance seulement, on observait la maîtrise du déplacement, de la sélection d'outils, du lancer, etc., y compris pour les néophytes : "Tout le monde a maintenant bien compris comment Minecraft fonctionne. La totalité des élèves est capable de se déplacer et de casser/récupérer/sélectionner des blocs" (animateur de l'atelier). Ce fonctionnement par niveaux leur a aussi permis de pratiquer la lecture et le respect de consignes formulées, c'est-à-dire de travailler leurs compétences méthodologiques. Il est à noter toutefois que la réalisation de ces niveaux n'était pas si "facile» et que c'est la persévérance et le travail d'équipe qui ont, pour beaucoup d'élèves, permis d'arriver au bout : "les niveaux sont quand même difficiles pour moi vu que j'ai jamais essayé». Ces niveaux ont finalement contribué à la stimulation de la motivation intrinsèque des élèves (Deci et Ryan, 1985) en les maintenant concentrés et engagés dans la tâche et en situation d'expérience optimale (Csikszentmihalyi, 1990). Cette situation d'expérience optimale est susceptible de participer au développement de certaines compétences « scolaires » chez les élèves. En effet, totalement investis dans le jeu et motivés par leur activité, ces derniers seraient plus ouverts aux apprentissages (Csikszentmihalyi, 1990).

\section{Plusieurs impacts « scolaires » observés}

Les données recueillies ont également permis de mieux comprendre comment la pratique observée du jeu Minecraft participait au développement de l'autonomie des élèves, comme l'indiquent eux-mêmes plusieurs élèves : «tu peux construire à ton rythme, c'est toi qui décides ce que tu construis puis moi c'est ça quejaime». 
En outre, la collaboration et l'entraide ont aussi été favorisées par la pratique de ce jeu, puisque près de 70\% des élèves affirment avoir été aidés par des amis (Figure 6) et $100 \%$ disent avoir déjà aidé un ami.

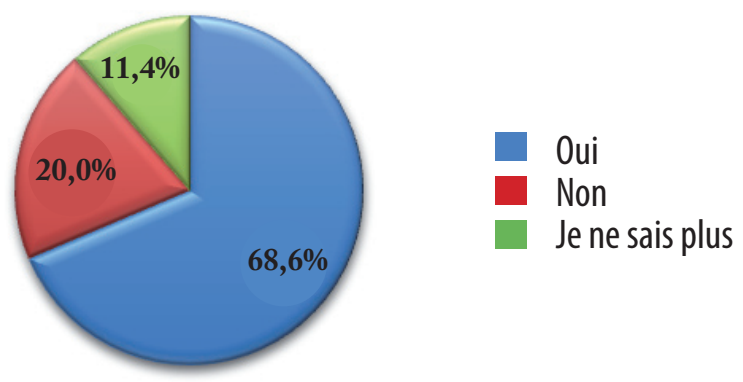

\section{Figure 6}

Pourcentage d'élèves qui ont été aidés par un ami en jouant à Minecraft.

L'animateur de l'atelier confirme d'ailleurs l'importance de la collaboration et de la coopération entre les élèves en avançant qu'il y a une "grande coopération entre les jeunes » et que «les nouveaux progressent plus vite ", ce qui est « sûrement dî au fait qu'ils soient avec d'autres plus avancés pour les aider ». Il s'agit donc ici d'une réelle entraide entre les élèves, ce qui a contribué à offrir une ambiance de groupe positive et à développer leurs habiletés sociales. Les données recueillies lors des entrevues individuelles révèlent également que la collaboration, l'entraide et le travail d'équipe sont des habiletés qui sont favorisées par la pratique du jeu Minecraft. Par exemple, lors des entrevues individuelles, lorsqu'il a été demandé aux élèves ce qu'ils faisaient lorsqu'il y avait un problème à résoudre, plusieurs des réponses illustraient l'importance du travail d'équipe, de la collaboration, etc. :

- «je demande à des amis qui sont plus capables que moi » et la majorité confirme :

- "J'apprends beaucoup à jouer en équipe "

- "le travail d'équipe c'est plus amusant»

- les autres "miaident beaucoup et je peux apprendre plus de choses"

- "si tu ne comprends pas, tu peux demander de l'aide à d'autres personnes"

- "dans Minecraft, on est plus ensemble, on est plus soudés, et on travaille beaucoup mieux en équipe que dans un autre travail»

- " quand j'ai un problème, souvent, j'essaie de chercher une solution en demandant à mes copains qu'est-ce qu'ils en pensent»

- " travailler en équipe c'est facile; être tout seul, ce sera pas facile»

- "je demande de l'aide à mon ami "

- "je demande à un ami à côté de moi et après il miaide"

C'est apparemment le fait de pouvoir s'amuser avec ses amis qui amène les élèves à procéder de même, parce que Minecraft «c'est comme une cour de récré, mais virtuelle» (élève). Ces conclusions rejoignent notamment celles de Nebel, Schneider et Rey (2016) pour qui l'impact d'un tel jeu venait, entre autres, de sa capacité à stimuler les activités collaboratives des élèves. 
Les données recueillies dans le cadre de cette recherche exploratoire révèlent que la pratique organisée du jeu Minecraft a profondément amélioré le sentiment de compétence des élèves ou encore leur estime de soi : "on dirait que je suis un pro et ils me demandent (sic) des questions que je connais". Les élèves ont également développé de meilleures compétences en communication orale : "on apprend à mieux communiquer entre nous» (élève). Par ailleurs, les données illustrent également comment Minecraft a permis de stimuler la créativité des élèves. En effet, ils ont ainsi pu concevoir de nombreux environnements, proposer des constructions et aménager des bâtiments d'une manière parfois particulièrement ingénieuse et de qualité : «Les élèves nèn restent pas moins créatifs » (animateur de l'atelier).

Les données recueillies révèlent même que cette créativité s'est accrue grâce à l'esprit concurrentiel développé entre les élèves : "On observe aussi de plus en plus de créativité grâce à la concurrence entre ces équipes» (animateur de l'atelier). Ils ont d'ailleurs particulièrement aimé construire des maisons (une des tâches à réaliser), ce que révèle l'enquête par questionnaire (Figure 7).

\begin{tabular}{l|c}
\hline Maison & $40 \%$ \\
\hline Titanic & $\mathbf{8 , 5 7 \%}$ \\
\hline Bateau & $\mathbf{5 , 7 1 \%}$ \\
\hline L'école & $\mathbf{5 , 7 1 \%}$ \\
\hline
\end{tabular}

\section{Figure 7}

Pourcentage de ce que les élèves ont préféré construire lors de l'atelier Minecraft.

En effet, lors des entrevues individuelles, les élèves ont aussi abondamment souligné l'aspect créatif inhérent à la pratique du jeu Minecraft :

- "il n'y a pas de limite dans l'imagination»

- «j'aime faire des constructions, je suis vraiment bon là-dedans, j’ai beaucoup d'imagination làdedans»

- "j’apprends à faire des objets, construire des objets », " tu peux faire ce que tu veux»

- "on peut mettre tout ce quion imagine»

- "on peut construire tout ce quion veut, on peut inventer quest-ce quion veut, créer des choses comme on peut inventer n'importe quoi quexiste pas dans la vérité»

- " on peut créer des choses»

- "on peut construire beaucoup d'affaires »

- "il n'y a vraiment aucune limite à ce quion peut faire "

Les élèves ont aussi développé des compétences en recherche d'information, particulièrement lorsqu'ils devaient se renseigner différentes manipulations à effectuer pour avancer dans les niveaux du projet 
Minecraft. Ils ont aussi progressé en résolution de problèmes : « suivre les niveaux leur apprenait à bien lire et comprendre un énoncé» (animateur de l'atelier). Enfin, lors des entrevues individuelles, plusieurs élèves ont indiqué que la pratique encadrée du jeu Minecraft les contraignait à "vraiment réfléchir » pour résoudre des problèmes, par exemple dans le cas où, pour passer à un niveau supérieur, il fallait qu'ils trouvent la façon de se procurer du charbon : "pour avoir du charbon, il faut résoudre un problème ».

Les données recueillies révèlent également que la pratique du jeu a permis aux élèves de respecter des suites logiques et ainsi d'appliquer un raisonnement inductif ou déductif en mathématiques. Ce constat est confirmé par l'animateur de l'atelier : "j’insiste aussi pour leur faire comprendre la suite logique des niveaux comme par exemple on leur fait construire un établi avant un four, car il faut un établi pour faire un four».

Dans l'une des tâches à réaliser, les élèves étaient amenés à apprendre diverses notions en agriculture, de même que certaines méthodes pour mieux faire pousser des plantes et élever des animaux (Figure 8). Cela leur a particulièrement plu, car, grâce à Minecraft, ils ont acquis plusieurs connaissances sur l'agriculture : «comme les plantes, quiest-ce quion a besoin pour les faire pousser » (élève).

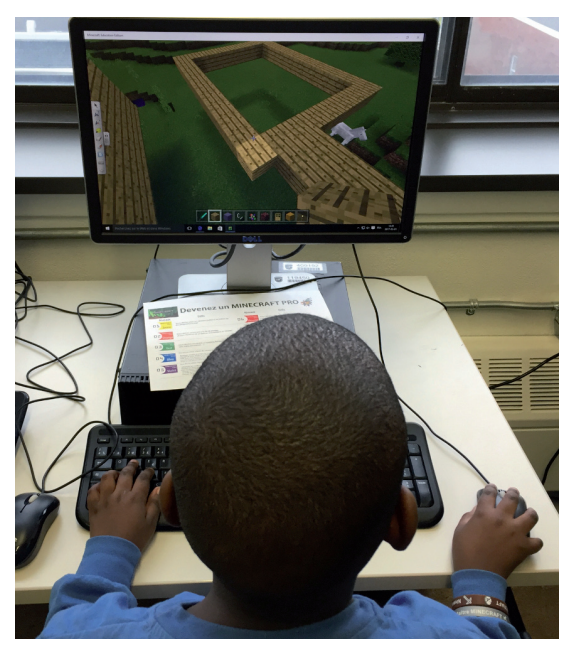

Figure 8

Un élève construisant un enclos.

La pratique du jeu Minecraft a également aidé les élèves à se repérer dans l'espace et à acquérir diverses notions en sciences, lorsqu' ils avaient, par exemple, trouvé les éléments permettant d'allumer un feu, faire fonctionner un train, etc. Selon les élèves, la pratique organisée du jeu Minecraft les a non seulement amenés à chercher l'information sur Internet, mais aussi à s'appliquer dans la recherche des solutions : "pour faire quelque chose, tu peux pas faire avec la rapidité, il faut que tu réfléchis (sic) et que tu te concentres pour faire des choses sur Minecraft ». Selon l'animateur, lorsque les élèves ne connaissent pas la réponse à un défi, ils vont développer des habiletés en recherche d'information et devenir meilleurs pour aller chercher "les informations sur les encyclopédies web, sur YouTube, ou sur les sites tels que Minecraft Wiki ", un wiki spécifiquement dédié à ce jeu vidéo. Les entrevues réalisées auprès des élèves confirment également que la pratique du jeu Minecraft les rend meilleurs dans la recherche d'information. 
- "je vais sur Internet, j'écris - comment construire un fort dans Minecraft-, je clique enter puis ça me le montre, et je revais (sic) sur Minecraft et je le fais »

- "je vais sur YouTube voir comment fabriquer"

- "la dernière fois je suis allé sur YouTube et j'ai construit une maison ».

Les observations vidéographiées réalisées dans le cadre de cette recherche exploratoire révèlent également que les élèves sont nombreux à avoir cherché comment effectuer telle ou telle manipulation sur YouTube. Les observations et les entrevues individuelles illustrent également que la pratique du jeu Minecraft a amené les élèves à faire attention à l'écriture (lorsqu'ils écrivaient, par exemple, sur divers panneaux de signalisation présents dans les mondes créés). L'écriture s'est aussi avérée importante pour nommer leur maison ou leur quartier. Enfin, ils ont souvent communiqué par écrit avec leurs camarades de classe durant la pratique du jeu, ce qui les a amenés à la fois à lire et à écrire, ce que confirment plusieurs entrevues individuelles réalisées avec les élèves : "on pratique notre orthographe, notre conjugaison en français ».

En cherchant à résoudre les défis posés dans les diverses tâches à réaliser dans le projet Minecraft, les élèves se sont également améliorés en anglais, car plusieurs ressources trouvées n’étaient pas en français, et les élèves devaient parfois aussi écrire certains mots en anglais. Cela a aussi été souligné par plusieurs élèves lors des entrevues: "connaître l'anglais était important $[. .$.$] pour savoir ce que ça veut dire le nom$ du bloc».

Les données recueillies révèlent également que la pratique structurée de ce jeu vidéo a permis aux élèves de développer leur persévérance : « la persévérance [...] la progression est constante » (animateur de l'atelier). La persévérance des élèves a aussi pu être identifiée lors des observations vidéographiées lorsque des élèves ont été surpris à recommencer à plusieurs reprises certains niveaux. Outre le plaisir pris à jouer, les élèves ont confié apprendre le travail en équipe (pour 82,4\% d'entre eux), la construction de " choses » $(82,4 \%)$, la créativité $(79,4 \%)$, l'informatique $(47,1 \%)$ et, même, les mathématiques $(32,4 \%)$ en jouant Minecraft (voir Figure 9). Cela va dans le sens des travaux de Overby et Jones (2015) qui mettaient en avant le fort impact du jeu sur la créativité des élèves.

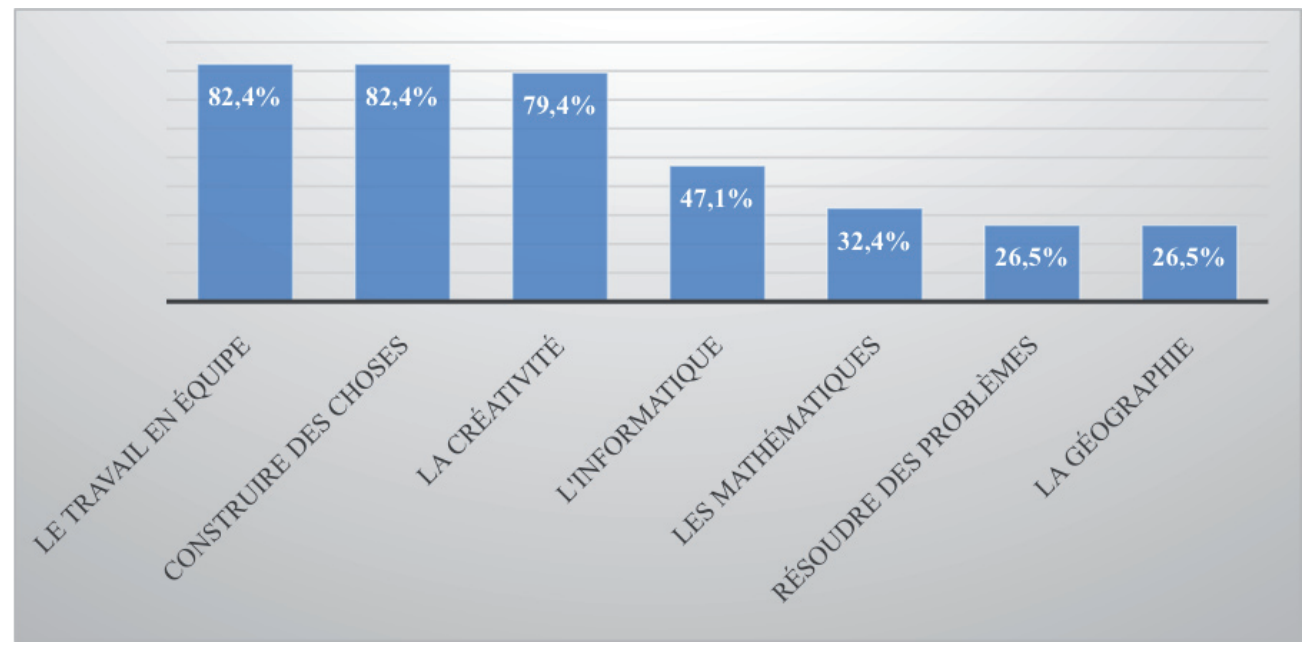

Figure 9

Pourcentage de ce qu’ont l'impression d'apprendre les élèves en jouant à Minecraft. 
Les données recueillies par l'entremise du questionnaire révèlent également que les élèves apprennent les mathématiques (périmètre, aire, etc.), l'informatique et la géographie par la pratique organisée du jeu Minecraft. Les entrevues réalisées confirment également ce constat.

- "ça peut miapprendre à bien compter, parce que pour construire il faut bien compter, parce que dans Minecraft il faut faire des constructions paires, il y a aussi des constructions impaires, mais c'est plus difficile »

- "j’apprends les mathématiques, aussi la géographie, le volume, les mesures pour savoir combien de blocs mettre»

- "les mathématiques si par exemple on dit: mets le tiers de la maison d'une couleur"

- "il faut que je calcule tout le nombre exact de blocs dont j'ai besoin »

Les élèves, les filles comme les garçons, ont aussi développé des compétences liées aux technologies de l'information et de la communication, en programmation informatique et en logique computationnelle, grâce notamment aux lignes de code qu'ils peuvent appliquer dans le jeu. Ils sont d'ailleurs près de $80 \%$ à avoir utilisé le code pour le passage de différents niveaux Minecraft. Cela constitue un progrès important par rapport à leur niveau initial sur Minecraft. Les entrevues réalisées auprès des élèves confirment d'ailleurs leurs progrès en programmation, notamment pour être en mesure de : «téléporter, comment mettre le jour, la nuit, comment enlever les méchants, comment mettre les méchants " (élève). Ce dernier point est particulièrement important, car il vient confirmer que les élèves seraient en mesure d'apprendre à coder grâce à la pratique du jeu vidéo Minecraft. Quand on connaît l'importance d'enseigner le codage aux élèves aujourd'hui (voir Karsenti et Bugmann, 2017), ce résultat prend une importance majeure dans cette intégration du numérique à l'école.

Enfin, les données recueillies révèlent que les élèves ont pu apprendre des éléments historiques, notamment lorsqu'ils créaient un monde datant d'il y a plusieurs centaines d'années. Cela les amenait à faire le niveau Minecraft $\mathrm{PRO}$ où les élèves avaient alors à créer des univers virtuels basés sur des faits géographiques ou historiques (construction du Titanic, de la tour Eiffel, du Forum Romain, etc.). Ils ont même eu l'impression de progresser au fur et à mesure des ateliers :

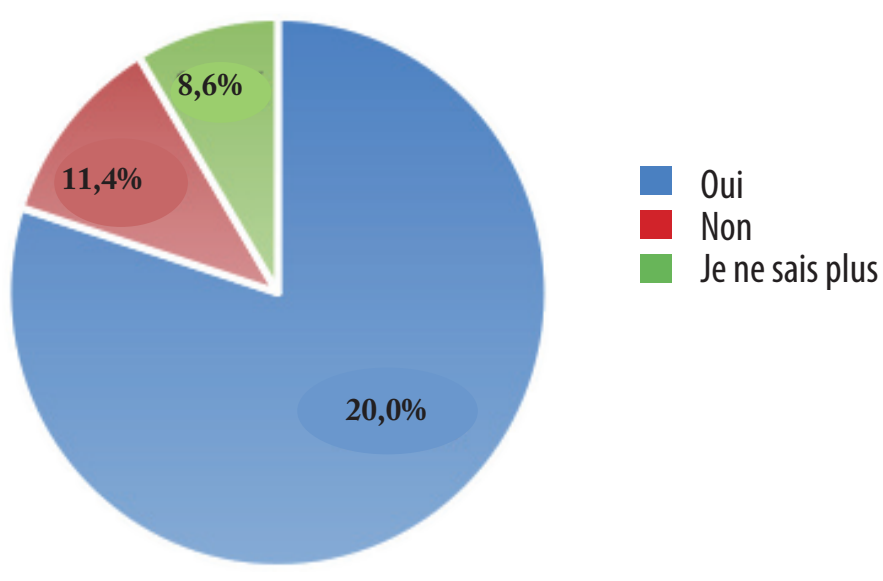

Figure 10

Pourcentage d'élèves qui ont l'impression d'avoir progressé à Minecraft à l'issue de l'atelier. 
Finalement, l'animateur confirme la survenue de potentiels apprentissages, y compris à plus long terme: "il y a des choses quion ne peut pas mettre en l'air sans que ça tombe donc ça instaure la gravité, certaines choses comme ça, ils ne s'en rendent même pas compte qu'ils l'apprennent, mais plus tard dans la vie ils se diront: "ah ben oui, ça c'est normal" ".

\section{Conclusion}

Dans un contexte où le jeu vidéo Minecraft est de plus en plus populaire dans les écoles, nous avons cherché à mieux comprendre ses avantages éducatifs auprès de 118 élèves du primaire. Les résultats de l'étude ont illustré le réel intérêt pédagogique de la pratique encadrée du jeu vidéo Minecraft en contexte scolaire. Les données analysées ont notamment permis de mettre en évidence plusieurs des avantages associés à la pratique encadrée de ce jeu à l'école, qui dépasse largement la simple motivation des élèves. Enfin, malgré les résultats positifs présentés dans notre étude, il semble important de rappeler que cette étude n'indique en aucun cas que la pratique libre du jeu vidéo peut être bénéfique. Nous avons, au contraire, pris soin de rappeler à de nombreuses reprises que les avantages que nous avons été en mesure d'observer semblent en tous points liés à une pratique pédagogique, structurée et encadrée de la pratique du jeu vidéo Minecraft à l'école. Notre étude ne portait donc pas sur les pratiques d'encadrement des élèves face aux dérives qui peuvent aussi faire partie de la pratique, parfois abusive, des jeux vidéo. Ainsi, malgré les impacts positifs présentés dans ce rapport, il importe de fournir aux élèves, ou aux enfants, un cadre dans leur pratique des jeux vidéo comme Minecraft, pour éviter certaines dérives. Un jeu vidéo qui revêt un grand potentiel éducatif comme Minecraft ne doit pas être synonyme d'absence de limites. Sans cadre, les enfants risquent de ne jamais vouloir arrêter de jouer. C'est pour cela notamment que, dans cette recherche exploratoire, nous avons fourni à la fois un contexte scolaire (les niveaux) et un encadrement (un animateur) pour l'usage de Minecraft à l'école. C'est d'ailleurs l'originalité de notre projet qui se voulait non seulement ludique, mais aussi et surtout viable d'un point de vue pédagogique. Aussi, en liant attentes pédagogiques de l'école et aspirations ludiques des élèves, tout en respectant la structure hiérarchisée de l'enseignement traditionnel, nous avons permis un rapprochement efficace entre le jeu vidéo et l'environnement « classe ».

Enfin, de façon générale, il est important de rappeler la nécessité de trouver un juste équilibre entre la pratique des jeux vidéo et d'autres activités. Entre obsession et outil à potentiel éducatif exceptionnel, il revient tant au parent qu'à l'enseignant de baliser l'usage de ce jeu pour que les jeunes gardent un juste équilibre entre des moments où les technologies sont utilisées, et d'autres où elles ne le sont pas.

\section{Notes}

1. https://fr.wikipedia.org/wiki/Liste des jeux vid\%C3\%A9o les plus vendus\#Classement g.C3.A9n.C3.A9ral

2. $\quad$ https://www.washingtonpost.com/lifestyle/kidspost/minecraft-spawns-classroom-lessons/2013/03/14/717aed6687b8-11e2-98a3-b3db6b9ac586 story.html

3. http://www.pcgamer.com/minecraft-becomes-a-compulsory-class-for-swedish-school//

4. $\quad$ http://www.lepoint.fr/invites-du-point/idriss-j-aberkane/le-jeu-video-est-la-premiere-industrie-culturelle-23-082014-1856039 2308.php

5. http://www.education.gouv.qc.ca/references/publications/resultats-de-la-recherche/detail/article/indices-dedefavorisation/ 
6. Pour Jaillet et Larose (2009), les traces informatiques sont « d'abord la manifestation des faits » (p. 18). Les traces permettraient avant tout au chercheur de mieux documenter ce que des participants ont fait dans des contextes technologiques.

7. http://www-01.ibm.com/software/analytics/spss/

8. https://www.surveymonkey.com/

9. http://provalisresearch.com/fr/produits/logiciel-d-analyse-qualitative/

\section{Références}

American Psychological Association. (2013). Publication manual of the American Psychological Association (6 ${ }^{\mathrm{e}}$ éd.). Washington, DC : American Psychological Association.

Baranowski, T., Baranowski, J., Cullen, K. W., Marsh, T., Islam, N., Zakeri, I., ... deMoor, C. (2003). Squire’s Quest! American Journal of Preventive Medicine, 24(1), 52-61. http://dx.doi.org/10.1016/S0749-3797(02)00570-6

Bebbington, S. et Vellino, A. (2015). Can playing Minecraft improve teenagers' information literacy? Journal of Information Literacy, 9(2), 6-26. http://dx.doi.org/10.11645/9.2.2029

Callaghan, N. (2016). Investigating the role of Minecraft in educational learning environments. Educational Media International, 53(4), 244-260. http://dx.doi.org/10.1080/09523987.2016.1254877

Cipollone, M., Schifter, C. C. et Moffat, R. A. (2014). Minecraft as a creative tool: A case study. International Journal of Game-Based Learning (IJGBL), 4(2), 1-14. http://dx.doi.org/10.4018/ijgbl.2014040101

Craft, J. (2016). Rebuilding an empire with Minecraft: Bringing the classics into the digital space. The Classical Journal, 111(3), 347-364. http://dx.doi.org/10.5184/classicalj.111.3.0347

Crombie, W., Moffat, D. C. et Shabalina, O. (2016, octobre). Video games can temporarily increase creativity; especially puzzle games. Communication présentée à la $10^{\text {th }}$ European Conference on Games Based Learning, Paisley.

Csikszentmihalyi, M. (1990). Flow: the psychology of optimal experience. New York, NY : Harper \& Row.

Deci, E. L. et Ryan, R. M. (1985). Intrinsic motivation and self-determination in human behavior. New York, NY : Plenum Press. http://dx.doi.org/10.1007/978-1-4899-2271-7

Dewey, J. et Deledalle, G. (1983). Démocratie et éducation : introduction à la philosophie de l'éducation. Lausanne : L'Âge d'Homme.

Ferguson, C. J. et Olson, C. K. (2013). Friends, fun, frustration and fantasy: Child motivations for video game play. Motivation and Emotion, 37(1), 154-164. http://dx.doi.org/10.1007/s11031-012-9284-7

Fonction publique de l'Ontario. (2016). Définir les compétences du 21e siècle pour l'Ontario. Compétences du 21'e siècle. Document de réflexion. Repéré à https://pedagogienumeriqueenaction.cforp.ca/wp-content/uploads/2016/02/Ontario-21stcentury-competencies-foundation-FINAL-FR AODA EDUGAINS Feb-19 16.pdf

Jaillet, A. et Larose, F. (2009). Le numérique dans l'enseignement et la formation : analyses, traces et usages. Paris : L'Harmattan.

Karsenti, T. et Bugmann, J. (2017). Les écoles canadiennes à l'heure du code? Éducation Canada, 57(1).

Komis, V., Depover, C. et Karsenti, T. (2013, 11 mars). L'usage des outils informatiques en analyse des données qualitatives. Adjectif. Repéré à http://www.adjectif.net/spip/spip.php?article216

L'Écuyer, R. (1990). Méthodologie de l'analyse développementale de contenu : méthode GPS et concept de soi. Québec, QC : Presses de l'Université du Québec.

Magnussen, R. et Elming, A. (2015). Cities at play: Children's redesign of deprived neighbourhoods in Minecraft. Dans R. Munkvold et L. Kolas (dir.), Proceedings of the 9th European Conference on Games Based Learning: ECGBL 2015 (p. 331-337). Reading : Academic Conferences and Publishing International. 
Méndez, M. D. C. L., Arrieta, A. G., Dios, M. Q., Encinas, A. H. et Queiruga-Dios, A. (2016). Minecraft as a tool in the teaching-learning process of the fundamental elements of circulation in architecture. Dans M. Graña, J. M. LópezGuede, O. Etxaniz, Á. Herrero, H. Quintián et E. Corchado (dir.), International Joint Conference SOCO'16-CISIS'16ICEUTE'16 (p. 728-735). Cham : Springer. http://dx.doi.org/10.1007/978-3-319-47364-2 71

Miles, M. B. et Huberman, A. M. (1991). Analyse des données qualitatives : recueil de nouvelles méthodes. Louvain-la-Neuve : De Boeck.

Ministère de l'Éducation et de l'Enseignement supérieur. (2017). Taux de décrochage annuel. Repéré à http://www.education. gouv.qc.ca/references/publications/resultats-de-la-recherche/detail/article/taux-de-decrochage-annuel/

Moffat, D. C., Crombie, W. et Shabalina, O. (2017). Some video games can increase the player's creativity. International Journal of Game-Based Learning (IJGBL), 7(2), 35-46. http://dx.doi.org/10.4018/IJGBL.2017040103

Morgan, M. L. (2015). Developing 21st century skills through gameplay: To what extent are young people who play the online computer game Minecraft acquiring and developing media literacy and the four Cs skills? (Thèse de doctorat). Accessible par ProQuest Dissertations \& Theses. (10020378)

Nebel, S., Schneider, S. et Rey, G. D. (2016). Mining learning and crafting scientific experiments: A literature review on the use of Minecraft in education and research. Journal of Educational Technology E' Society, 19(2), 355-366.

Overby, A. et Jones, B. L. (2015). Virtual LEGOs: Incorporating Minecraft into the art education curriculum. Art Education, 68(1), 21-27.

Piaget, J. (1959). La formation du symbole chez l'enfant : imitation, jeu et rêve, image et représentation ( $2^{\mathrm{c}}$ éd.). Neuchâtel : Delachaux et Niestlé.

Picard, M. (2016). Les enjeux esthétiques du jeu vidéo : entre art, stylistique et interactivité. Sciences du jeu, (6).

Pusey, M. et Pusey, G. (2015). Using Minecraft in the science classroom. International Journal of Innovation in Science and Mathematics Education, 23(3). Repéré à https://openjournals.library.sydney.edu.au/index.php/CAL/article/view/10331

Ringland, K. E., Wolf, C. T., Faucett, H., Dombrowski, L. et Hayes, G. R. (2016). « Will I always be not social? »: Reconceptualizing sociality in the context of a Minecraft community for autism. Dans Proceedings of the 2016 CHI Conference on Human Factors in Computing Systems (p. 1256-1269). New York, NY : ACM. http://dx.doi.org/10.1145/2858036.2858038

Riordan, B. C. et Scarf, D. (2016). Crafting minds and communities with Minecraft. F1000Research, 5, 2339. http://dx.doi.org/10.12688/f1000research.9625.2

Roussel, K. (2017). Les protocoles verbaux (think-aloud protocols) : enjeux méthodologiques de validité pour la recherche en contexte scolaire. CJNSE/RCJCÉ, 8(1). Repéré à https://journalhosting.ucalgary.ca/index.php/cjnse/article/ view/30805

Shaftel, J., Pass, L. et Schnabel, S. (2005). Math games for adolescents. Teaching Exceptional Children, 37(3), 25-30. https://doi.org/10.1177/004005990503700304

Thorsteinsson, G. et Niculescu, A. (2016). Pedagogical insights into the use of Minecraft within educational settings. Studies in Informatics and Control, 25(4), 507-516. http://dx.doi.org/10.24846/v25i4y201612

Trudel, L., Simard, C. et Vonarx, N. (2006). La recherche qualitative est-elle nécessairement exploratoire? Recherches qualitatives, (5), 38-55. Repéré à http://www.recherche-qualitative.qc.ca/documents/files/revue/hors serie/hors serie v5/trudel.pdf

Winnicott, D. W. (1975). Jeu et réalité : l'espace potentiel. Paris : Gallimard.

\section{Pour citer cet article}

Karsenti, T. et Bugmann, J. (2018). Quels apports éducatifs du jeu vidéo Minecraft en éducation? Résultats d'une recherche exploiratoire menée auprès de 118 élèves du primaire. Formation et Profession, 26(1), 89-108.

http://dx.doi.org/10.18162/fp.2018.459 